\title{
SELENIO TOTAL Y VIABILIDAD CELULAR EN LA OBTENCIÓN DE SELENIO-LEVADURA
}

\author{
${ }^{*}$ Carmen Alicia Rodríguez Besta ${ }^{\mathrm{a}, \mathrm{c}}$, Mario Ceroni Galloso ${ }^{\mathrm{a}}$, \\ Elvito Fabián Villegas Silva ${ }^{\mathrm{b}}$, Teresa Dámaris Rebaza Cárdenas ${ }^{\mathrm{d}}$
}

\begin{abstract}
RESUMEN
Se obtiene selenio-levadura mediante el cultivo de la levadura Saccharomyces cerevisiae LALVIN Bourgovin RC 212 en el medio YEPD, con la adición de selenito de sodio (en concentraciones de 2 a $10 \mathrm{mg} \mathrm{Se}(\mathrm{IV}) / \mathrm{L}$ ) durante la fase de crecimiento exponencial, después de 48 horas a $30^{\circ} \mathrm{C}$ y $200 \mathrm{rpm}$. La cuantificación de selenio total intracelular en la seleniolevadura es factible con el método espectrofotométrico almidón-yoduro a $590 \mathrm{~nm}$ y la adición de $8 \mathrm{mg} \mathrm{Se}(\mathrm{IV}) / \mathrm{L}$ proporciona mayor biomasa, una viabilidad celular superior al $90 \%$, coloración rosada y un contenido de selenio total intracelular de $738 \pm 30 \mathrm{mg} / \mathrm{kg}$ en base seca. Finalmente, la metodología utilizada para determinar el porcentaje de viabilidad celular y el método analítico propuesto para cuantificar el selenio total intracelular, son alternativas accesibles para el control del bioproceso.
\end{abstract}

Palabras clave: selenio total, selenio-levadura, selenito, viabilidad celular.

\section{TOTAL SELENIUM AND CELULAR VIABILITY FOR OBTAINING SELENIUM-YEAST}

\begin{abstract}
Selenium-yeast is obtained by the culture of Saccharomyces cerevisiae LALVIN Bourgovin RC 212 in the medium YEPD, with sodium selenite addition (concentrations of 2 to 10 $\mathrm{mg} \mathrm{Se}(\mathrm{IV}) / \mathrm{L}$ ) during exponential growth phase, after 48 hours at $30^{\circ} \mathrm{C}$ and $200 \mathrm{rpm}$. Total intracellular selenium quantification in the selenium-yeast is feasible with the starch-iodine spectrophotometric method at $590 \mathrm{~nm}$ and the addition of $8 \mathrm{mg} \mathrm{Se}(\mathrm{IV}) / \mathrm{L}$ yields the most biomass, a $>90 \%$ cellular viability, pink coloration and a total intracellular selenium of $738 \pm 30 \mathrm{mg} / \mathrm{kg}$ in dry basis.

${ }^{a}$ Unidad de Posgrado de la Facultad de Química e Ingeniería Química, Universidad Nacional Mayor de San Marcos. Lima 01

${ }^{\text {b }}$ Departamento Académico de Química. Facultad de Ciencias, Universidad Nacional Agraria La Molina. Lima 12

c. Laboratorio de Química Biológica y Bioanálisis. Departamento Académico de Química. Facultad de Ciencias, Universidad Nacional Agraria La Molina. carb@la molina.edu.pe

d Laboratorio de Micología y Biotecnología "Marcel Gutiérrez Correa". Universidad Nacional Agraria La Molina. Lima 12.
\end{abstract}


Finally, the used methodology to find the cellular viability and the analytical method proposed to determine the total intracellular selenium are accessible for monitoring the bioprocess.

Key words: total selenium, selenium-yeast, selenite, cellular viability

\section{INTRODUCCIÓN}

El crecimiento de la levadura Saccharomyces cerevisiae en un medio de cultivo al que se adiciona selenito (selenización), permite obtener suplementos dietarios de selenio-levadura que suministran selenio biológicamente disponible en forma segura. El selenio, elemento esencial para la nutrición humana y animal, puede ser naturalmente acumulado por la levadura, razón por la cual, se utilizan las células de levadura en la producción de alimentos e insumos en las industrias farmacéuticas y biotecnológicas ${ }^{1}$.

El proceso de incorporación de selenio involucra el control de factores como la cantidad de selenito $\left(\mathrm{SeO}_{3}{ }^{2-}\right)$ o $\mathrm{Se}$ (IV) adicionado al medio de cultivo, la viabilidad celular de la biomasa ${ }^{2}$ y la cantidad de selenio incorporado al medio intracelular de la levadura, entre otros ${ }^{3}$. Adicionalmente, otros investigadores ${ }^{4}$ indican que al incrementar la cantidad de $\mathrm{Se}(\mathrm{IV})$ en el medio, se incrementa la inhibición del crecimiento celular de la levadura, lo que coincide con una mayor concentración de selenio total en las células de levadura.

Según Kieliszek et $a l .{ }^{5}$, las especies de levadura pueden mostrar diferente tolerancia al selenio y el efecto inhibitorio del selenio sobre el crecimiento de los microorganismos puede basarse en la aparición de estrés oxidativo en las células de levadura. Estos investigadores explican que el selenio puede actuar como un factor fisiológico capaz de modificar el crecimiento de las células de levadura, por eso conocer su influencia en el crecimiento de la levadura puede contribuir a mejorar la producción de la biomasa enriquecida con selenio.

Aunque la medida de la densidad óptica es una forma rápida y fácil de estimar el crecimiento de la biomasa, determinar la viabilidad celular de la levadura es un ensayo accesible, que además permite estimar la condición de la población celular, pues una disminución en la viabilidad celular indica inhibición del crecimiento y la inducción de la respuesta al estrés ${ }^{6}$.

Respecto a la cuantificación del contenido de selenio total en muestras de selenio-levadura, generalmente, se realiza mediante una técnica espectrofotométrica o electroquímica, después de un proceso de digestión /disolución completa de la matriz ${ }^{7}$.

El objetivo en este trabajo es cultivar la levadura Saccharomyces cerevisiae con la adición de 2 a $10 \mathrm{mg} \mathrm{Se(IV)/L} \mathrm{al} \mathrm{medio} \mathrm{de} \mathrm{cultivo,} \mathrm{para} \mathrm{obtener} \mathrm{selenio-levadura,} \mathrm{determinar}$ espectrofotométricamente el selenio total intracelular y evaluar ese contenido respecto a la viabilidad celular de la biomasa, para suministrar métodos de cuantificación accesibles en el control del bioproceso. 


\section{PARTE EXPERIMENTAL}

\section{Material biológico}

Levadura seca activa, Lalvin RC 212 (Bourgovin, GMO and gluten free). Saccharomyces cerevisiae cerevisiae producida por Lallemand INC. Montreal Qc, Canada HIW 2N8.

\section{Estándar}

SELM-1 (material de referencia certificado de levadura enriquecida con selenio).

\section{Reactivos}

Selenito de sodio P.A. (99 \%) Panreac AppliChem; HCl Baker ACS (36,5-38 \%), $\mathrm{HNO}_{3}$, $\mathrm{HClO}_{4}$, yoduro de potasio (99\%) Merck Emsure, Almidón soluble Scharlau.

\section{Equipos}

Autoclave ALP modelo CLG-32L/40M/40L, cabina de bioseguridad Biobase modelo 11231BBC86 MSC Clase II, agitador orbital Thermo Scientific MAXQ 7000, espectrofotométro Biomate 3 Thermo Electron Corporation, pHmetro UB-10 Denver Instrument, microscopio Olympus modelo CX31RBSFA, cámara Neubauer- BOECO Germany, bright line, modelo Tiefe Depth. Profondeur $\left(0.100 \mathrm{~mm}-0.0025 \mathrm{~mm}^{2}\right)$, centrífuga Sorvall Legend Thermo Scientific, congeladora Frigidaire, espectrofotométro Biomate 3 Thermo Electron Corporation, espectrofotómetro $10 \mathrm{~S}$ Genesys.

\section{Metodología}

\section{Preparación del medio de cultivo}

La preparación del medio de cultivo líquido YEPD (yeast extract, peptone, dextrose) y de la disolución de selenito de sodio (conteniendo $1000 \mathrm{mg} \mathrm{Se}(\mathrm{IV}) / \mathrm{L}$ ), las condiciones de esterilización así como la medida del $\mathrm{pH}$ del medio de cultivo se realizaron según lo descrito por Rodríguez et al. ${ }^{2}$.

\section{Preparación del inóculo}

Se pesaron 0,011 g de levadura seca activa, LALVIN Bourgovin RC 212, que se adicionaron a $50 \mathrm{~mL}$ de YEPD estéril, en un matraz erlenmeyer de $250 \mathrm{~mL}$. El matraz se colocó en baño maría a $36 \pm 1^{\circ} \mathrm{C}$ durante 20 minutos, después se retiró, se mezcló el YEPD con la levadura y se continuó con la agitación a $30^{\circ} \mathrm{C}$ y 200 rpm durante 24 horas $^{8}$.

\section{Adición de selenito}

En la cámara de bioseguridad, se adicionaron $5 \mathrm{~mL}$ de inóculo (cultivo de 24 horas de

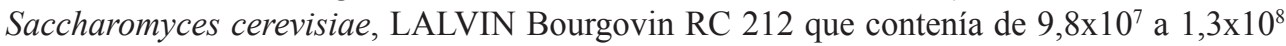
$\mathrm{cel} / \mathrm{mL})^{8}$ a cada matraz erlenmeyer de $250 \mathrm{~mL}$ conteniendo $45 \mathrm{~mL}$ de medio líquido YEPD estéril. Después de inocular, los matraces debidamente acondicionados se colocaron en el agitador a $30^{\circ} \mathrm{C}$ y $200 \mathrm{rpm}$. Transcurridas 5 horas de crecimiento (fase de crecimiento exponencial), se agregaron volúmenes de disolución de selenito de sodio en todos los medios experimentales, excepto en uno (control), con el propósito de obtener concentraciones de 0 (control), 2, 4, 6, 8 y $10 \mathrm{mg} \mathrm{Se(IV)/L.} \mathrm{Posteriormente,} \mathrm{se} \mathrm{continuó} \mathrm{la} \mathrm{agitación} \mathrm{de} \mathrm{todos} \mathrm{ellos}$ a $30^{\circ} \mathrm{C}$ y $200 \mathrm{rpm}$ hasta completar 48 horas de cultivo. Se realizaron cuatro repeticiones. 


\section{Densidad óptica y viabilidad celular}

Se realizaron cuatro repeticiones de las mediciones de densidad óptica (DO) y de recuento celular, la DO se realizó a $600 \mathrm{~nm}$ y el recuento celular con tinción ${ }^{2}$ para cada una de las concentraciones de $\mathrm{Se}(\mathrm{IV}) / \mathrm{L}$ a las 48 horas.

La viabilidad celular alcanzada en los cultivos se calcula como $\%$ de viabilidad celular $=$ [(número de células viables $/ \mathrm{mL} \div$ número de células totales $/ \mathrm{mL}$ ) x 100].

\section{Recuperación de biomasa de levadura}

Los cultivos de levadura en la fase estacionaria (48 horas) se colocaron en tubos falcón de $50 \mathrm{~mL}$ de capacidad, luego se separó la biomasa de levadura del sobrenadante (medio de cultivo) mediante centrifugación a $4^{\circ} \mathrm{C}$ y $4000 \mathrm{x} \mathrm{g} \mathrm{rpm} \mathrm{durante} 10$ minutos 9 . Después de la separación, la biomasa de levadura recuperada se colocó a $-20^{\circ} \mathrm{C}$.

\section{Preparación de estándares}

En la preparación directa de estándares de selenio, se pesaron 0,0139 gramos de selenito de sodio, se llevaron a $2 \mathrm{~mL}$ con agua ultrapura y a partir de esa disolución se obtuvieron por dilución disoluciones estándar de 100 y $20 \mathrm{mg}$ Se(IV)/L, respectivamente. También se realizaron adiciones de selenito de sodio a levadura sin selenio de la siguiente forma: a 0,0137 gramos de selenito de sodio se adicionaron $0,0400 \mathrm{~g}$ de levadura seca, luego se agregaron $20 \mu \mathrm{L}$ de agua ultrapura, $150 \mu \mathrm{L}$ de $\mathrm{HClO}_{4}\left(70 \%\right.$ p/p) y $50 \mu \mathrm{L}$ de $\mathrm{HNO}_{3}(54 \% \mathrm{p} / \mathrm{p})$, se sometieron a calentamiento moderado, luego se agregaron $50 \mu \mathrm{L}$ de $\mathrm{HCl} 6 \mathrm{M}$ y nuevamente se calentó, enfrió y neutralizó en frío con $100 \mu \mathrm{L}$ de $\mathrm{NaOH} 6 \mathrm{M}$ para llevar a un volumen de $2 \mathrm{~mL}$. Después se agitó, centrifugó y el residuo se resuspendió con $1,5 \mathrm{~mL}$ de agua ultrapura. A partir de esta suspensión, se realizaron diluciones de 100 y $20 \mathrm{mg} \mathrm{Se(IV)/L,} \mathrm{para} \mathrm{proseguir}$ con el procedimiento general de determinación espectrofotométrica de selenio total.

Para el análisis del material de referencia certificado SELM-1, se pesaron 0,0600-0,0800 g, se agregaron $40 \mu \mathrm{L}$ de agua ultrapura, $300 \mu \mathrm{L}$ de $\mathrm{HClO}_{4}(70 \% \mathrm{p} / \mathrm{p})$ y $100 \mu \mathrm{L}$ de $\mathrm{HNO}_{3}$ $(54 \% \mathrm{p} / \mathrm{p})$, se sometieron a calentamiento moderado, luego se agregaron $100 \mu \mathrm{L}$ de $\mathrm{HCl} 6 \mathrm{M}$ se calentó, enfrió y neutralizó en frío con $200 \mu \mathrm{L}$ de $\mathrm{NaOH} 6 \mathrm{M}$ para llevar a un volumen de $4 \mathrm{~mL}$, luego se mezcló, centrifugó y el residuo se resuspendió en 3,0 $\mathrm{mL}$ de agua ultrapura. Después se realizó el procedimiento general de la determinación espectrofotométrica ${ }^{8}$.

\section{Preparación de muestra de selenio-levadura}

Se pesaron $0,200 \mathrm{~g}$ de biomasa de selenio-levadura en suspensión (conservada a $-20^{\circ} \mathrm{C}$ ), luego se realizó el lavado con $2 \mathrm{~mL}$ de agua ultrapura, se mezcló en el vortex, posteriormente se aplicó ultrasonido (20 kHz por un minuto), se centrifugó a $3500 \mathrm{rpm}$ por cinco minutos y se eliminó el sobrenadante. Al sedimento se adicionaron $150 \mu \mathrm{L}$ de $\mathrm{HClO}_{4}(70 \% \mathrm{p} / \mathrm{p})$ y 50 $\mu \mathrm{L}$ de $\mathrm{HNO}_{3}(54 \% \mathrm{p} / \mathrm{p})$, se sometieron a calentamiento, después se agregaron $50 \mu \mathrm{L}$ de $\mathrm{HCl}$ $6 \mathrm{M}$ se calentaron, enfriaron y neutralizaron en frío con $100 \mu \mathrm{L}$ de $\mathrm{NaOH} 6 \mathrm{M}$ completando a $1,5 \mathrm{~mL}$ con agua destilada, se agitó, centrifugó y el residuo se resuspendió en $1,5 \mathrm{~mL}$ de agua ultrapura, se midieron $300 \mu \mathrm{L}$ de la solución y a continuación se realizó la determinación espectrofotométrica de selenio total ${ }^{8}$. 


\section{Determinación espectrofotométrica de selenio total}

La determinación espectrofotométrica almidón-yoduro de selenio total intracelular de selenio-levadura se fundamentó en la siguiente yodometría: $\left(\mathrm{SeO}_{3}\right)^{2-}+2 \mathrm{I}^{1-}+6 \mathrm{H}^{1+} \rightarrow \mathrm{Se}^{0}+$ $\mathrm{I}_{2}+3 \mathrm{H}_{2} \mathrm{O}$.

El procedimiento general consistió en medir un volumen de estándar o de muestra al que se adicionaron $5 \mathrm{~mL}$ de almidón al 0,1\%(p/v), 0,5 $\mathrm{mL}$ de EDTA al $5 \%(\mathrm{p} / \mathrm{v}), 0,5 \mathrm{~mL}$ de KI al $2 \%(\mathrm{p} / \mathrm{v})$ y $0,5 \mathrm{~mL}$ de $\mathrm{HCl} 6 \mathrm{M}$, luego se dejaron reaccionar durante 10 minutos, se completó con agua destilada hasta $10 \mathrm{~mL}$, se homogeneizó y se realizó la medida de absorbancia a 590 $\mathrm{nm}$ antes de transcurridos 15 minutos $^{8}$.

\section{RESULTADOS Y DISCUSIÓN}

\section{Densidad óptica y viabilidad celular}

Las medidas de densidad óptica y porcentaje de viabilidad celular de la levadura, después de 48 horas de cultivo en el medio YEPD ( $\mathrm{pH}$ 6), a las concentraciones de Se(IV) evaluadas, se encuentran en la tabla 1 donde se aprecia que los valores de DO, así como los de viabilidad celular, disminuyen conforme es mayor el contenido de $\mathrm{Se}(\mathrm{IV})$ en el medio de cultivo. También se observa que la magnitud de las disminuciones son más evidentes en los valores de viabilidad celular, a partir de la adición de $6 \mathrm{mg} \mathrm{Se}(\mathrm{IV}) / \mathrm{L}$, lo que sugiere que probablemente se produce mayor inhibición del crecimiento a las concentraciones de 8 y $10 \mathrm{mg} \mathrm{Se}(\mathrm{IV}) / \mathrm{L}$, considerando que las adiciones se realizaron en todos los casos durante la fase de crecimiento exponencial de la levadura Saccharomyces cerevisiae LALVIN Bourgovin RC 212.

Tabla 1. DO y Viabilidad celular de la selenio-levadura a las 48 horas de cultivo.

\begin{tabular}{ccc}
\hline $\begin{array}{c}\text { Se(IV) adicionado } \\
(\mathrm{mg} / \mathrm{L})\end{array}$ & DO & $\begin{array}{c}\text { Viabilidad celular } \\
(\%)\end{array}$ \\
\hline 0 & 2,084 & 99,4 \\
2 & 2,032 & 99,3 \\
4 & 1,978 & 99,2 \\
6 & 1,959 & 99,4 \\
8 & 1,952 & 93,6 \\
10 & 1,863 & 87,3 \\
\hline
\end{tabular}

\section{Determinación espectrofotométrica de selenio total}

En la figura 1 se encuentra la curva estándar para concentraciones de 0 a $0,3 \mathrm{mg} \operatorname{Se}(\mathrm{IV}) / \mathrm{L}$, la ecuación es $\mathrm{y}=0,46 \mathrm{x}-0,02$ y el $\mathrm{R}^{2}=1$; mientras que la curva estándar para el rango de concentraciones entre 0 a $1,2 \mathrm{mg} \operatorname{Se}(\mathrm{IV}) / \mathrm{L}$ tiene la ecuación $\mathrm{y}=0,411 \mathrm{x}-0,012$ y un $\mathrm{R}^{2}=$ 0,999 , como se observa en la figura 2 .

Respecto a las recuperaciones para los estándares de selenito de sodio, se obtuvieron valores de $99,98 \pm 0,10 \%$ para $20 \mathrm{mg} \mathrm{Se}(\mathrm{IV}) / \mathrm{L}$ y $99,99 \pm 0,08 \%$ para $100 \mathrm{mg} \mathrm{Se}(\mathrm{IV}) / \mathrm{L}$, mientras que 
en los estándares preparados con adición de selenito de sodio a la levadura seca (sin selenio),

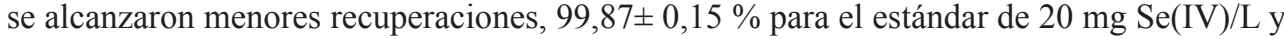
$99,90 \pm 0,11 \%$ para el estándar de $100 \mathrm{mg} \mathrm{Se(IV)/L.}$

En general, las altas recuperaciones obtenidas se explican por el cuidadoso proceso de digestión ácida o mineralización desarrollado, para lograr la oxidación completa de la materia orgánica, la oxidación completa a Se(VI) y luego la reducción a Se(IV).

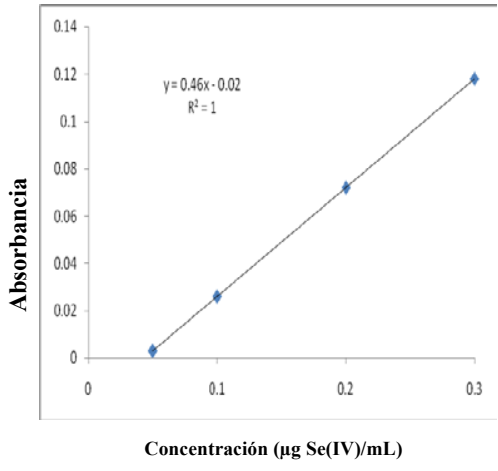

Figura 1. Curva estándar para selenio por el método almidónyoduro a $590 \mathrm{~nm}$. Concentraciones de 0 a $0,3 \mu \mathrm{g} \operatorname{Se}(\mathrm{IV}) / \mathrm{mL}$.

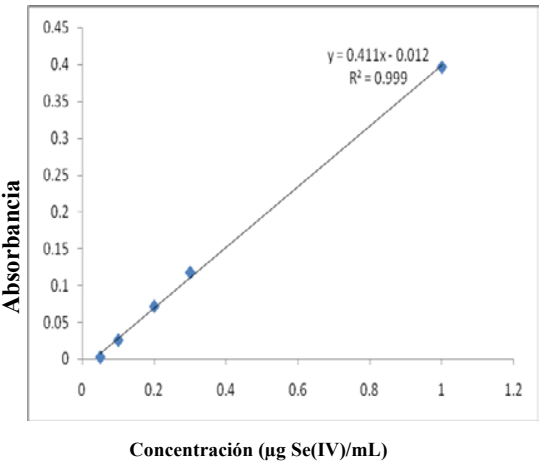

Figura 2. Curva estándar para selenio por el método almidónyoduro a $590 \mathrm{~nm}$. Concentraciones de 0 a $1,2 \mu \mathrm{g} \mathrm{Se}(\mathrm{IV}) / \mathrm{mL}$.

El resultado del análisis de selenio total $(n=3)$ para el material de referencia certificado (MRC) de levadura selenizada, SELM-1, es de $2010 \pm 50 \mathrm{mg} \mathrm{Se} / \mathrm{kg}$ en base seca, lo que

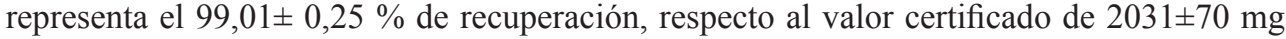
$\mathrm{Se} / \mathrm{kg}$ en base seca. Este resultado indica el buen desempeño del método espectrofotométrico almidón-yoduro a $590 \mathrm{~nm}$ en la cuantificación de selenio total en el SELM-1.

\section{Selenio total en selenio-levadura}

Las muestras de biomasa de selenio-levadura mantienen su morfología celular después de tres lavados con agua ultrapura y aplicación de ultrasonido, lo que representa una alternativa para eliminar Se(IV) extracelular remanente de la pared celular de la levadura.

La etapa de digestión ácida o mineralización de las muestra de selenio-levadura en suspensión requiere un procedimiento cuidadoso y progresivo, pues el ácido perclórico no es un oxidante en disolución acuosa, debido a que forma una mezcla azeotrópica con el agua, que hierve a $170^{\circ} \mathrm{C}$, temperatura a la cual es un oxidante poderoso y rápido ${ }^{10}$, lo que implica que primero se deben eliminar los vapores nitrosos, luego proseguir con la acción del ácido perclórico hasta oxidación total del selenio intracelular a Se(VI) y luego la reducción cuantitativa a $\mathrm{Se}(\mathrm{IV})$ con la adición de ácido clorhídrico ${ }^{11}$. 
Los resultados de selenio total en el medio intracelular de las muestras de levadura control y selenizadas, provenientes de los crecimientos a las diferentes concentraciones de Se(IV), se observan en la tabla 2, donde se aprecia que la cantidad de selenio total intracelular se incrementa conforme es mayor la cantidad de Se(IV) adicionado al cultivo, a partir de 4 y en mayor magnitud para 6, 8 y $10 \mathrm{mg} \mathrm{Se}(\mathrm{IV}) / \mathrm{L}$ (figura 3) tal como indican Ponce et al. ${ }^{4}$.

Tabla 2. Selenio total intracelular en selenio-levadura.

\begin{tabular}{cc}
\hline $\begin{array}{c}\text { Se(IV) } \\
\text { adicionado } \\
(\mathrm{mg} / \mathrm{L})\end{array}$ & $\begin{array}{c}\text { Selenio total } \\
(\mathrm{mg} / \mathrm{kg})\end{array}$ \\
\hline 0 & 0,2 \\
2 & $170 \pm 8$ \\
4 & $177 \pm 8$ \\
6 & $402 \pm 17$ \\
8 & $738 \pm 30$ \\
10 & $990 \pm 65$ \\
\hline
\end{tabular}

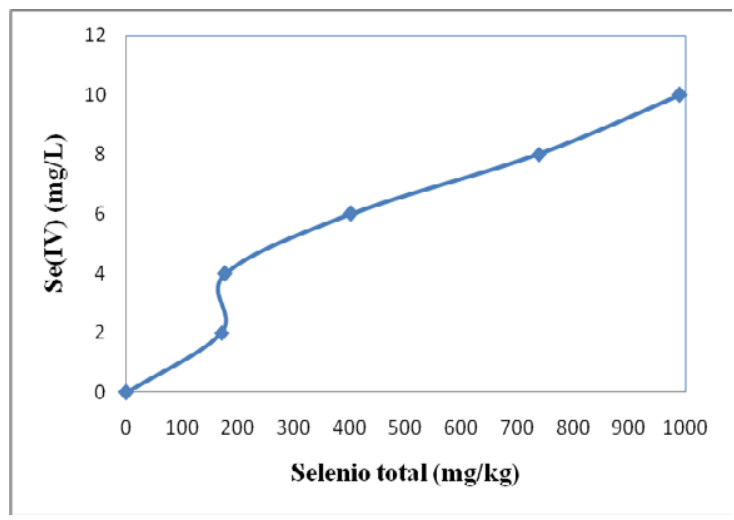

Figura 3. Selenio total intracelular en selenio- levadura en función de la adición de Se(IV).

Además, en la figura 4 se observa que a menores valores de DO corresponden mayores contenidos de selenio total intracelular, tendencia que también se aprecia para el porcentaje de viabilidad celular en la figura 5. Al comparar ambas tendencias, con las mismas adiciones de Se(IV) al medio de cultivo de la levadura (tabla 2), se encuentra que los valores de DO son cercanos a 2,10 y la viabilidad celular cercana al $100 \%$ cuando la cantidad de selenio total es menor a $200 \mathrm{mg} / \mathrm{kg}$ de selenio total intracelular. Sin embargo, para concentraciones superiores a $400 \mathrm{mg} / \mathrm{kg}$ de selenio total intracelular, los valores del porcentaje de viabilidad celular descienden más drásticamente que los de DO, lo que implica que la levadura ha incorporado selenio mediante sus procesos metabólicos, con la consiguiente conversión a formas orgánicas de selenio, aunque también es probable que mayores concentraciones de selenio total intracelular se manifiestan en estrés oxidativo ${ }^{5}$ que coincide con la inhibición del crecimiento y acumulación de selenio elemental. 


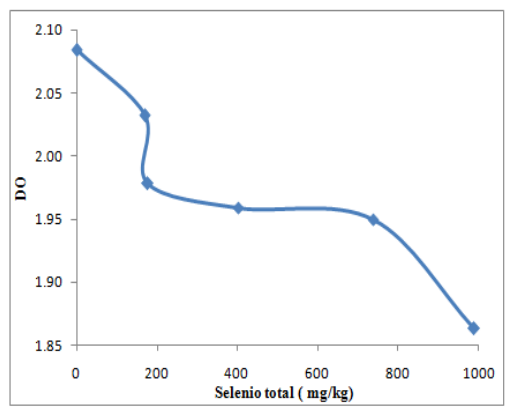

Figura 4. Selenio total y DO de las muestras de selenio-levadura.

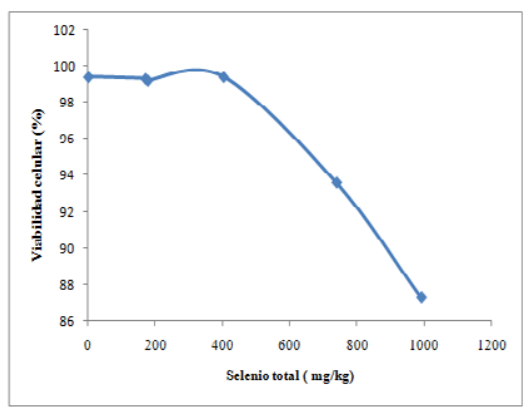

Figura 5. Selenio total y viabilidad celular de las muestras de seleniolevadura.

La incorporación de selenio descrita coincide con lo reportado por Kieliszek et al. ${ }^{9}$, respecto a que un menor tiempo de exposición y baja concentración de Se(IV) en el medio de cultivo es el método más efectivo para evitar la acumulación de selenio en la biomasa de levadura.

Por otro lado, las adiciones de selenito al YEPD en concentraciones de 8 y $10 \mathrm{mg} \mathrm{Se}(\mathrm{IV}) / \mathrm{L}$, durante la fase de crecimiento exponencial de la levadura, producen mayor biomasa cuando la levadura Saccharomyces cerevisiae LALVIN Bourgovin RC 212 se cultiva a $30^{\circ} \mathrm{C}$ y 200 rpm durante 48 horas, aunque el porcentaje de viabilidad celular es de 93,6 \% y 87,3\%, respectivamente, con una biomasa con coloración rosada en ambos casos, lo es un indicativo de un nivel intermedio de acumulación de selenio ${ }^{3}$.

\section{CONCLUSIONES}

De acuerdo a las evidencias disponibles, cultivar la levadura Saccharomyces cerevisiae LALVIN Bourgovin RC 212 en el medio YEPD, adicionando selenito de sodio a las 5 horas de la inoculación (fase de crecimiento exponencial), a concentraciones entre 2 y 10 $\mathrm{mg} \mathrm{Se}(\mathrm{IV}) / \mathrm{L}$, en las condiciones descritas, permite obtener selenio-levadura. Además, es factible la cuantificación de selenio total intracelular mediante el método espectrofotométrico almidón-yoduro a $590 \mathrm{~nm}$, aplicado luego de un proceso de digestión ácida.

Finalmente, las metodologías utilizadas para determinar el porcentaje de viabilidad celular y el contenido de selenio total intracelular, son alternativas accesibles, que suministran información sobre la inhibición del crecimiento y la acumulación de selenio en la levadura, para el control del bioproceso. 


\section{AGRADECIMIENTO}

Los autores expresan su agradecimiento a la Ph.D. Gretty Villena Chávez, Directora del Laboratorio de Micología y Biotecnología "Marcel Gutiérrez Correa", por las facilidades brindadas para la ejecución de ensayos de recuento de levaduras. Al Vicerrectorado de Investigación de la Universidad Nacional Agraria La Molina, en el marco del Convenio MINEDU-UNALM 2017, por el financiamiento otorgado para el desarrollo de la presente investigación.

\section{REFERENCIAS BIBLIOGRÁFICAS}

1. Kieliszek M, Blazejak S, Gientka L, Bzducha-Wrobel A. Accumulation and metabolism of selenium by yeast cells. Appl Microbiol Biotechnol. 2015; 99: 5373-5382.

2. Rodríguez C, Cjuno J, Ceroni M. Influencia de la concentración de Se(IV) en la viabilidad celular durante el crecimiento de la levadura Saccharomyces cerevisiae. Rev Soc Quim Perú. 2018; 84(2): 228-238.

3. Marinescu G, Stoicescu AG. Industrial nutrient medium use for yeast selenium preparation. An Univ "Dunarea Jos" Galati Fasc VI. 2011; 35(1):45-53.

4. Ponce de León CA, Bayón MM, Paquin C, Caruso JA. Selenium incorporation into Saccharomyces cells: a study of different incorporation methods. J Appl Microbiol. 2002; 92:602-610.

5. Kieliszek M, Blazejak S, Bzducha-Wróbel A, Kot AM. Effect of selenium on growth and antioxidative system of yeast cells. Mol Biol Rep. 2019; 46(2):1797-1808. doi: 10.1007/ s11033-019-04630-z

6. Jamnik P, Raspor P. Methods for Monitoring Oxidative stress Response in Yeasts. J. Biochem Mol Toxicol. 2005; 19 (4): 195-203.

7. Ochsenkuhn-Petropoulou M, Tsopelas F. Speciation Analysis Especially of Tin and Seleniuin in Environmental Matrices. En Michalke B. Metallomics: Analytical Techniques and Speciation Method. Weinheim: Wiley-VCH Verlag GmbH; 2016. P. 131-151.

8. Rodríguez Best CA. Cuantificación cromatográfica y electrométrica de selenometionina y selenocisteína intracelular de levadura Saccharomyces cerevisiae en función de la concentración de selenito en el medio de cultivo. [Tesis doctoral]. Lima: Universidad Nacional Mayor de San Marcos; 2019.

9. Kieliszek M, Blazejak S, Placzek M. Spectrophotometric evaluation of selenium binding by Saccharomyces cerevisiae ATCC MYA-2200 and Candida utilis ATCC9950 yeast. J Trace Elem Med Biol. 2016; 35:90-96.

10. Feigl F, Anger V. Pruebas a la Gota en Análisis Inorgánico. México D.F.: El Manual Moderno; 1980.

11. Svarc-Gajic' JV, Suturovié ZJ, Marjanovic NJ, Kravic Z. Chronopotentiometric Stripping Analysis of Selenium in Feed: Development of a Method. Asian J Anim Vet Adv. 2006; 1(1):13-22. 